\title{
Sentinel Lymph Node Biopsy with Axillary Lymph Node Dissection
}

National Cancer Institute

\section{Source}

National Cancer Institute. Sentinel Lymph Node Biopsy with Axillary Lymph Node

Dissection. NCI Thesaurus. Code C47854.

A surgical procedure in which the sentinel node is removed followed by dissection and removal of the axillary node group. 